\title{
De quelques paramètres de la souveraineté
}

Parameters of Sovereignty

\section{Michel Izard}

\section{OpenEdition}

\section{Journals}

Édition électronique

URL : http://journals.openedition.org/span/875

DOl : $10.4000 /$ span. 875

ISSN : 2268-1558

\section{Éditeur}

École pratique des hautes études. Sciences humaines

\section{Édition imprimée}

Date de publication : 1 décembre 1990

Pagination : 69-92

ISSN : 0294-7080

\section{Référence électronique}

Michel Izard, «De quelques paramètres de la souveraineté », Systèmes de pensée en Afrique noire [En ligne], 10 | 1990, mis en ligne le 25 juin 2013, consulté le 02 mai 2019. URL : http:// journals.openedition.org/span/875; DOI : 10.4000/span.875 
par

\section{Michel Izard}

Nous avons longuement insisté ailleurs sur l'existence de deux registres sémantiques de la souveraineté chez les Moose du Yatenga'. Le premier s'organise autour de la notion de naam, "pouvoir", le second autour de celle de panga, "force".

Le terme naam rend compte à la fois du concept et de sa réalisation: du "pouvoir" en général et de ses manifestations comme avatars institutionnels. Un naam, c'est un commandement ou une "chefferie", à quelque niveau hiérarchique qu'on l'envisage. Sans entrer dans le complexe détail des modalités de détention du naam, on distinguera trois types de "chefferie"?: 1) la chefferie "royale" (fonction souveraine); 2) les chefferies locales (fonctions de commandement); 3 ) les chefferies de cour (fonctions de service). Qui dit "chefferie" dit "chef"; le mot rendu par "chef", naaba, est dérivé directement de

\footnotetext{
"Le Yatenga (Yaad(ga) tenga, "pays de (Naaba) Yaad(ga)", du nom du fondateur de la dynastie en place depuis le milieu du XV ${ }^{\prime \prime}$ siècle) est l'un des royaumes du Moggo (Burkina Faso), pays des Moose, sing. Mooga, dont la langue est le moore. Les thèmes évogués dans cet article $I^{\prime}$ ont été dans plusieurs publications antérieures, notanment dans Izard, 1985.

${ }^{2}$ La tripartition proposée ici est à distinguer de celle qu'opère le pouvoir central parmi les détenteurs du naam, en distinguant les gens de cour ou naa(ba) tiir(i) demba ("gens de la maison du chef"), les "maîtres de la guerre" ou tâ(po) sobnamba, représentants des dynasties moose antérieures à celle du Yatenga et des plus anciennes couches de chefs du Yatenga, et les nakombse, membres du lignage royal du groupe de descendance dynastique du Yatenga.
} 
naam, puisqu'il en est le pluriel (le pluriel de naam, nanamba ou nanamse, a donc valeur de double pluriel de naam). Tout détenteur d'un naam est un naaba; formellement, la réciproque est vraie - tout naaba est le détenteur d'un naam -, même si, pratiquement, l'usage du mot naaba dépasse largement le cadre de la détention d'un naam pour pointer le statut, même occasionnel, de quelqu'un qui est le premier, qui est "devant", qui, dans le sens le plus trivial du terme, "commande". Le mode de construction du vocabulaire de la titulature fondée sur la détention d'un naam associe le mot naaba et le naam particulier en cause. A la tripartition des formes de naam donnée cidessus correspond ainsi une répartition comparable des chefs, parmi lesquels on distinguera donc: 1) le chef du Yatenga ou Yatenga naaba, c'est à dire le roi ; 2) les chefs des unités locales de commandement ${ }^{3}$ : ainsi, le chef de l'unité de commandement de Gursi porte-t-il le titre de Gursi naaba, etc.; 3) les premiers des "chefs" de cour, dont les titres associent le terme naaba à un mot qui évoque la nature de la fonction de cour correspondante: le togo naaba, "chef de la parole", est le héraut du roi, le balum naaba, "chef de la salutation", est le responsable de la vie du palais, le weranga naaba, "chef de l'aire aux chevaux", a sous ses ordres les palefreniers royaux, etc. Les deux premiers types de détention du naam renvoient à deux modes d'appréhension de l'espace de souveraineté: le territoire comme tout (le Yatenga) et comme ensemble de ses parties constituantes (les unités de commandement). Le tout n'est pas équivalent à la somme des parties. En effet, l'espace du royaume est le support de deux trames de divisions territoriales dont les frontières intérieures ne coïncident pas et dont une seule sature cet espace. Le premier de ces deux systèmes est celui qui subdivise l'espace entier du royaume en ce que par commodité nous appelons des "unités de maîtrise de la terre", qui

\footnotetext{
${ }^{3}$ L'emploi sans précaution du mot français "village" pour traduire le moore tenqa (cf. cidessous, n. 4) peut conduire à de fâcheuses confusions dès qu'il s'agit d'envisager le mode de découpage du territoire du royaume permettant de rendre compte autant de l'assise territoriale du pouvoir des chefs que de l'autorité des maîtres de la terre: nous parlons respectivement d' "unité de commandement" et d"unité de maîtrise de la terre".
} 
sont autant d'obédiences relevant de la maîtrise matérielle et spirituelle du sol par des lignages fondateurs de villages appartenant en principe à la couche réputée autochone de la population, celle des "gens de la terre" (teng demba) ou "fils de la terre" (teng biise), en place dans le pays à l'arrivée des conquérants moose. Il n'est aucune partie de l'espace du royaume qui ne relève d'une unité de maîtrise de la terre, chacune d'entre elles étant marquée par la présence: d'un quartier fondateur de village, d'un autel de la terre (tenga) et d'un "maître de la terre" (teng $(a)$ soba ${ }^{4}$, doyen du quartier fondateur et sacrificateur sur l'autel de la terre. Le second des deux systèmes évoqués ci-dessus est celui des "unités de commandement", qui relèvent du pouvoir mooga et qui ont à leur tête un "chef" (naaba) ou plus précisément un "chef de village" (teng naaba), le terme tenga ayant par métonymie - passage du sol à la population qui l'occupe -, le sens de "village". Le système des unités de commandement ne sature pas l'espace du royaume: en d'autres termes, si tous les villages ont en principe un maître de la terre, tous n'ont pas un chef, de telle sorte qu'il y a plusieurs dizaines de villages qui sont "commandés" par un maître de la terre, qui n'entretient aucun lien institutionnel, de quelque nature qu'il soit, avec le pouvoir royal, auquel sont par contre strictement inféodés les chefs locaux. La bipartition de la société du royaume (à laquelle n'appartiennent pas les Peuls qui vivent sur le territoire du royaume) entre "gens du pouvoir" (les descendants des conquérants moose) et "gens de la terre" (les autochtones) correspond à l'existence de deux régimes d'autorité, le premier qui concerne les hommes, le second qui concerne la terre. L'une des plus fondamentales fonctions du roi est d'être le garant de "l'alliance" entre le pouvoir et la terre.

\footnotetext{
"Le mot tenga désigne la "terre", à la fois comme instance ordonnatrice de la fertilité du sol et de l'intégrité du groupe et come personnalisation de cette instance (Napaaga Tenga); également (au plur. têse, comme au sing.), l'autel sur lequel on sacrifie à la terre, l'officiant étant le tengsoba; enfin l'unité territoriale correspondant à l'obédience d'un tenqsoba et par extension 1 'unité d'habitat et de population qui l'occupe: en ce sens, tenga peut être rendu dans le langage courant par "village".
} 
Que dans le contexte du vocabulaire de la souveraineté, naam "pouvoir" - fasse système avec panga - "force" - est admirablement exprimé par l'énoncé: "le pouvoir reçoit, la force prend". Les deux notions rendent compte de deux aspects de toute souveraineté: qu'elle se donne pour/se manifeste comme à la fois productrice de violence ("quand la force suit le chemin, le bon droit coupe à travers la brousse") et inductrice d'un consensus ("c'est le sujet qui fait le chef"). Le souverain est à la fois naaba, "chef", et pang(a) soba, "maître de la force". Dans pangsoba (comme dans tengsoba, cf. ci-dessus), le substantif soba, "celui qui détient", "celui qui a la maîtrise de", est construit sur le verbe so, dont est également dérivé le substantif solem, qu'on ne peut se contenter de traduire, comme on l'a fait trop souvent, par "commandement", au sens territorial du terme, alors que sa signification est beaucoup plus large, en référence précisément à la signification de so: le solem de quelqu'un, c'est ce qui est sous son autorité, sous son contrôle, c'est ce qu'il détient, ce dont il a la maîtrise. La dualité de la souveraineté ainsi mise en évidence retentit sur la distinction entre chefs locaux et chefs de cour. Les chefs locaux sont et sont seulement des "chefs", voués à la détention du naam sous la forme d'un naam particulier. Les chefs de cour ne sont pas envisagés comme des "chefs" au sens premier du terme, mais, à la fois, comme des "gens de la force" ( $p a ̂ s(e)$ demba, expression dans laquelle le pluriel de panga, pâse, introduit une nuance de respect et de crainte) et comme des "personnes dignes d'estime" (neba somba, plur. de neda soma, contracté en nesomba, dont le sing. propre est nesomde): on retrouve à ce niveau institutionnel la double détermination de la violence et du consensus.

Il s'agit maintenant d'établir comment les positions de naaba et de pangsoba font système avec celle de rima, à laquelle correspond par excellence la figure du souverain. Le mot rima a pour radical le verbe ri, qui désigne l'action d'absorber un aliment qui ne se mâche pas, ce qui renvoie à la fois - et de manière connexe - à la croyance générale en l'existence d'une séquence secrète du rituel d'acquisition du statut de rima consistant en l'absorption d'un breuvage dont il est 
au moins dit qu'on y a plongé le second des regalia du Yatenga', le naam tiido, et à l'usage métaphorique du verbe $r i$ en place de "commander". Sur ri sont formés rita, "celui qui règne", que l'on retrouve dans des constructions comme naa(ba) rita et $k u(m)$ rita (cf. ci-dessous), et ringu, "royaume". Ce même verbe entre en composition avec naam dans rinaam, qui désigne à la fois le pouvoir du roi et la royauté. Tout nouveau chef, y compris le "chef" du Yatenga, une fois qu'il a été choisi par l'instance politique ad hoc, acquiert le titre de naaba après avoir été intronisé. La cérémonie d'intronisation est identique pour tous les chefs, à ceci près qu'alors que c'est le souverain qui préside à l'intronisation des chefs locaux et des chefs de cour, c'est le premier des chefs de cour qui préside à celle du souverain. Le souverain est donc d'abord "chef" (naaba); une seconde intronisation va le faire "roi" ( $r i m a)$; par contre, l'acquisition du titre de pangsoba n'est pas expressément ratifiée par quelque manifestation que ce soit: la seule chose que l'on puisse dire à cet égard, c'est qu'au moment où le roi devient rima, il dispose ipso facto de la pleine maîtrise de la "force". Dès lors, deux hypothèses distinctes et partiellement exclusives l'une de l'autre peuvent être introduites. La première serait que l'acquisition ritualisée du statut de rima et celle, apparemment non ritualisée, du statut de pangsoba, sont une seule et même chose. La seconde serait que le futur rima acquiert la maîtrise de la force avant d'être intronisé rima, de telle manière que cette acquisition serait une condition nécessaire et suffisante d'accession au statut de rima. Nous tenterons ici de valider la seconde hypothèse, c'est-à-dire de montrer que la "force"- panga intervient dans le processus de passage du statut royal initial au statut royal final comme élément médiateur entre le "pouvoir" au sens général du terme - naam - et le "pouvoir royal" - rinaam -.

\footnotetext{
S A la royauté du Yatenga sont associés trois regalia majeurs, qui sont, par ordre d'importance: le naam wubri, le naam tiido et le naam gango; les deux premiers sont des emblemes permanents de la dynastie régnante, le troisième est renouvelé à chaque intronisation de rima. Le naam wubri, du nom du fondateur de la dynastie régnante de Wogdgo (Ouagadougou), Naaba Wubri, est un objet qui n'est jamais présenté en public, au contraire du naam tiido (on ne peut mieux traduire tiido que par "chose").
} 
Le sacre (l'intronisation du rima) est la manifestation culminante d'un voyage qui conduit le souverain de la localité royale où résidait son prédécesseur (et où il a été intronisé naaba) à celle où il a choisi de résider ${ }^{\epsilon}$, en passant par la localité résidentielle du fondateur de la dynastie, Tangazugu', où se déroule la cérémonie qui transforme le naaba en rima. L'ensemble de ce périple est appelé ringu, mot dont on vient de voir qu'il signifie "royaume". Le ringu comprend deux parties. La première, qui conduit le roi de la localité royale où il a été nommé à Tangazugu, revêt un double aspect: 1) par le moyen d'une suite de sacrifices qui sont effectués sur des autels de la terre des localités royales, actuelles et anciennes, qui constituent le "domaine royal"s, le nouveau roi renouvelle l'alliance du pouvoir et de la terre; 2) dans le même temps, d'étape en étape, il acquiert toute une série de signes de souveraineté: interdits alimentaires, ustensiles individuels, premier cheval cérémoniel, porteurs de regalia, etc. Le roi arrive à Tangazugu en situation de pouvoir être intronisé rima. Au départ de Tangazugu commence la seconde partie du ringu, qui fait systématiquement contraste avec la première: le futur roi en quête de l'onction de la terre est devenu pleinement roi, celui qui, pendant la première partie de son voyage, a "donné" (des cadeaux en nature, des femmes, dont les destinataires sont principalement les maîtres de

\footnotetext{
6 Le Yatenga n'a pas de capitale: en principe, un roi peut résider où bon lui semble, à quelques impossibilités ou interdictions près. En fait, le développement du service royal, à compter du début du XVIII* siècle, a entrainé la création de localités royales. A la fin du $X_{I X}{ }^{*}$ siècle, on comptait quatre résidences royales ou naa(ba) têse, sing. naa tenqa, "village du chef", les anciennes résidences royales ayant statut de kiims(e) têse, sing. kiims tenca, "village des ancêtres". Depuis. 1892, tous les rois ont résidé dans un seul des quatre natêse (plur. de naatenga) résidentiels, Waiguyo (Ouahigouya).

${ }^{7}$ Le fondateur de la dynastie actuelle du Yatenga, Naaba Yaadga, a d'abord résidé près du village de Gursi, dans le sud-cuest du Yatenga actuel, au lieu dit Tangazugu, le "sormet de la colline", aujourd'hui importante fraction du commandement local de Gursi. C'est à Tangazugu qu'a lieu le sacre, les principales manifestations se déroulant autour de la "pierre du pouvoir" (naam kugri) et sur le seuil occidental de l'ancienne demeure de Naaba Yaadga, le Yikeende (yii(ri) keende, "vieille maison").

"Par "domaine royal", expression gue les Moose rendent par naa(ba) solem, nous entendons l'ensemble des localités relevant directement de l'autorité du roi: naatêse et kiimstêse (cf. ci-dessus, $n .6$ ) en font notamment partie.
} 
la terre ordonnateurs des sacrifices faits sur les autels de la terre), commence à "recevoir"; c'est au cours de cette seconde partie que le roi honore la mémoire de ses ancêtres par des sacrifices effectués sur les tombes du cimetière royal ${ }^{9}$ et, au même endroit, reçoit l'hommage des membres de la branche du lignage royal à laquelle il appartient ${ }^{10}$ et celui de tous les dignitaires moose du royaume. A suivre notre hypothèse, ce serait pendant la première partie du ringu que le futur rima se rendrait progressivement maître de la "force", par la médiation de la "terre" (tenga, personnifiée en Napaaga Tenga, où naa (ba) paaga signifie "épouse de chef"). Le pouvoir et la terre fondent ensemble la légitimité du pouvoir du roi, que valident à la fois l'ancestralité du pouvoir - la suite des rois - et l'autochtonie liée au monde de la terre, et ce serait par l'instauration de sa relation à la terre que le "pouvoir" deviendrait "force".

Le souverain, en tant que naaba, dispose d'un pouvoir dont ni la légitimité ni la suprématie n'est contestable: on rappellera sur ce point que le plus fameux des rois du Yatenga, Naaba Kango (1757-1787), n'a pas fait le ringu. Le passage du statut de naaba à celui de rima transforme cependant radicalement le statut du souverain. De cette mutation, on donnera les trois principales conséquences. La première est qu'à sa mort, le roi-rima est enterré dans le cimetière royal, ce qui vaut intégration à l'ancestralité dynastique et assurance pour

\footnotetext{
"Le naa(ba) yaado est le cimetière (yaado, "tombes", sing. yaogo) des rimnamba depuis la première moitié du XVIIe siècle; il est situé près du village de Somnyaa, ancienne résidence ropale, où a lieu, au cours de la deuxième phase du ringu une grande salutation du roi (naa(ba) pusum).

10 La généalogie du groupe de descendance dynastique du Yatenga est stratifiée en branches constituées par les descendants, génération après génération, des rois en situation d'aîné sur la généalogie dynastique: il suit qu'un roi fils aîné et son fils aîné n'appartiennent pas à la mêne branche, puisque chacun d'eux est à l'origine d'une branche, identifiée par son nom. Les cinq dernières branches constituent le lignage rogal; la formation d'une nouvelle branche entraîne automatiquement la sortie du lignage royal de la branche la plus ancienne. Tous les Moose, ou membres du Moos(e) buudu (cf. note 12), ont le statut de nakombse, c'est-à-dire de descendants de chefs qui ne sont pas devenus chefs, mais, du point de vue du pouvoir central, dans chaque royaume, ne sont considérés corme des nakonbse que les membres du lignage royal, qui constituent donc une aristocratie de sang, les autres Moose étant, dans cette même perspective, des gens du cormun, zemba ou talse.
} 
"l'ancêtre" (kiima) qu'est devenu le roi défunt d'être honoré chaque année, à l'occasion des fêtes royales qui ouvrent la nouvelle année. La seconde est que les fils du roi-rima peuvent prétendre au trône, ce qui n'est pas le cas pour les fils d'un roi seulement demeuré naaba. La généalogie dynastique, dont la stratification en branches commande le processus mécanique de sortie des membres du lignage royal génération après génération, est uniquement une généalogie de rimnamba (plur. de rima). Cette règle de transmission du pouvoir royal propose une formulation restrictive de l'une des deux règles générales qui commandent les modalités de transmission du pouvoir chez les Moose: celle en vertu de laquelle il faut être fils de chef pour pouvoir devenir chef " . La troisième réside en une sacralisation de la personne royale, qui se manifeste principalement par l'observance de nombreuses prescriptions et interdits dont l'effet est d'occulter pour le plus grand nombre tout ce qui, dans la vie quotidienne du souverain, relève de déterminations d'ordre intime: il s'agit de masquer la personne par le personnage. Bien que notre propos ne soit pas de traiter de ce problème, il convient d'insister sur le fait que cette sacralisation n'est nullement un confinement dans une sorte de ritualisation généralisée: on ne doit pas oublier que le roi est un "chef" et le "maître de la force".

L'opération qui consiste à passer de la trame d'ensemble de l'univers généalogique de la royauté à une généalogie dynastique ne prenant en compte que les seuls rimnamba introduit de la discontinuité là où l'idéologie mooga conçoit une continuité par delà le moment de l'irruption du naam dans l'histoire, qui procède d'une coupure. Les Moose se donnent pour instance créatrice et ordonnatrice du monde une divinité masculine, de nature céleste, personnifiée sous le nom de Naaba Wende, de sorte que ce dieu est d'emblée envisagée comme un "chef", dont l'"épouse" est Napaaga Tenga, la terre comme support

\footnotetext{
"Chez les Moose, la transmission du pouvoir obéit généralement à deux règles, qui ne surdéterminent pas l'identité du successeur d'un chef: 1) il faut être fils de chef pour être candidat à la chefferie; 2) le pouvoir passe d'aĩné à cadet: de "frère aîné" en "frère cadet", de "père" en "fils".
} 
de sacralité. Naaba Wende dispose par principe d'un naam dont procèdent tous les autres naam: le Wend(e) naam ou wendnaam. Il y a solution de continuité entre le wendnaam divin et le naam humain, ce qui n'est que le rappel de la transcendance de la figure divine. Par contre, le naam humain, véhiculé par une transmission agnatique, est substantiellement identique à quelque niveau d'exercice du pouvoir qu'on l'envisage. Le naam comme pouvoir en général existe indépendemment des formes de son instauration pratique, qui relèvent seules d'un discours hiérarchique. Ce caractère du naam d'être étranger à quelque accumulation ou déperdition que ce soit a sa traduction rituelle dans l'unicité de forme des intronisations à la fonction de naaba et, plus simplement, dans l'usa-ge général du titre même de naaba.

Tout se passe donc comme si la nécessité institutionnelle de créer au coeur du monde du pouvoir un statut qui soit radicalement autre que celui de naaba ait entrainé la nécessité congrue d'opérer une rupture rituelle totale entre les accessions aux statuts de naaba et de rima, le second des deux étant d'abord radicalement autre que le premier plutôt que supérieur à lui. La distinction entre les statuts de naaba et de rima ne nous semble pas seulement avoir pour effet de circonscrire le monde des rois au sein de celui des chefs, lui-même pris dans le système récurrent d'inclusions qui conduit, pour un royaume donné, du lignage royal à la généalogie d'ensemble du Moogo ${ }^{12}$. Elle nous paraît en outre surdéterminer le caractère irréductiblement singulier du roi en ouvrant et en fermant son règne par des rituels qui nous rappellent que la solution de continuité entre deux règnes successifs doit être réduite, sans que cet aspect de la mise en oeuvre de ces rituels en épuise la signification.

\footnotetext{
32. Tous les Moose sont censés être les descendants en ligne agnatique d'un ancêtre unique; toutes les généalogies dynastiques du Moogo, anciennes et actuelles, royales, régionales et locales, s'inscrivent sur la généalogie du groupe de descendance maximal de l'ancêtre fondateur, le Moos buudu, où buudu a le sens de patri-groupe de descendance.
} 
Considérons le Yatenga naaba au terme du ringu: il est naaba, pangsoba et rima, l'emphase institutionnelle et rituelle mise sur ce dernier statut indiquant bien que c'est en tant que rima que le souverain totalise les déterminations qui le définissent. Celles-ci n'épuisent cependant pas la "composition" de la personne royale, qui est d'abord une personne humaine (neda) comme les autres. A sa mort, cette personne humaine libère un ancêtre (kiima) que rien ne distingue de l'ancêtre d'un individu ordinaire, et, dans les funérailles royales, nous ne trouvons aucun élément qui donnerait à l'ancestralité royale quelque spécificité que ce soit, si l'on réserve la question de la vigilance rituelle qui en préserve la pérennité et, si l'on peut dire, la prospérité. Pour ce qui concerne la personne vivante du roi, le diagnostic par les rituels est tout autant négatif: les relations que le roi établit avec ses ancêtres propres ne se distinguent en rien de celles qu'établit n'importe quel homme. Force est de conclure qu'il n'existe pas d'articulation substantielle entre la personne humaine du roi et la personne royale en sa triple détermination.

La personne humaine en général est "produite" par une partition de la substance ancestrale qui est opérée par les "génies" de la brousse (kinkirse) ${ }^{13}$. En analysant la double relation: du pouvoir à l'autochtonie, d'une part, du pouvoir à l'ancestralité, d'autre part, nous avons montré que ces trois éléments:

\section{pouvoir/autochtonie/ancestralité}

formaient une triade qui faisait sémantiquement trame pour d'autres configurations ternaires, notamment:

$$
\text { personne/génie/ancêtre }
$$

et

identité/étendue/durée.

C'est en référence à ce résultat que nous allons examiner le mode de relation pouvant exister entre les déterminations naaba, pangsoba et rima. Notre hypothèse première, dans ce contexte, est que les

${ }^{13}$ Cf. notamment Izard, 1983 et 1986. 
paires naaba/pangsoba et naam/panga peuvent être transcrites en la métaphore d'une double valence royale. Si tel était le cas, le statut de rima serait bien une totalisation et formerait à cet égard, avec les statuts de naaba et de pangsoba une triade homologue de celles qui ont été évoquées ci-dessus.

Pour qu'il y ait dualité, il faut et il suffit, nous semble-t-il, que les deux éléments liés par la supposée relation duelle soient impliqués dans des processus dont la déconstruction produise deux séries comparables d'éléments secondaires s'opposant terme à terme. Le rappel de la succession des événements qui prennent place entre l'annonce de la mort du roi ${ }^{14}$ et la nomination de son successeur nous donne matière à poursuivre l'analyse dans le sens qui a été indiqué ci-dessus.

Les deux moments qui bornent la période de transition entre deux règnes successifs déterminent une durée de quatorze jours, qui est divisée en deux grandes séquences ayant chacune une durée de sept jours. Les sept premiers jours sont consacrés à la célébration des funérailles royales, le septième jour étant celui de l'inhumation dans le cimetière royal. Pendant cette première semaine - généralement d'un samedi à un vendredi -, le roi défunt est censé continuer de régner: la salutation quotidienne au roi des quatre premiers dignitaires de la cour a lieu devant l'entrée de la chambre où repose le cadavre du roi. Dans le même temps, le palais et la localité résidentielle vivent sous la menace permanente d'exactions perpétrées par les captifs royaux, qui sont par excellence des "gens de la force". Tout se passe donc comme si le naam et le panga se survivaient à eux-mêmes, respectivement par le simulacre de la salutation au roi et par l'irruption d'une violence sans contrôle, qui se manifeste par le pillage des habitations des épouses royales et des éventaires du marché. Entre le "pouvoir" et la "force", il n'y a pas équilibre, mais au contraire déséquilibre, au profit de la force. Au début de la seconde semaine, trois événe-

\footnotetext{
in Les Moose utilisent la semaine arabe de sept jours. La mort du roi prend effet à compter de son annonce officielle, dont le jour est choisi en fonction de la vocation exclusive de certains jours de la semaine à servir de cadre à tel ou tel type de manifestation.
} 
ments majeurs ont lieu, dont deux seront d'abord examinés: les nominations de la naapoko et du kurita.

Chez les Moose, à la mort d'un chef, l'intérim du pouvoir est assuré par l'aînée des filles du défunt, qui porte le titre de naa(ba) poko, "chef-femme". La naapoko, qui est généralement une femme d'un certain âge, quitte sa demeure pour celle de son père: pendant toute la durée de son maintien en fonction, son mari ne doit pas la voir. La naapoko prend la place de son père; elle en porte les vêtements, en imite les intonations et la démarche et agit à sa place, elle "est" son père. Cette règle générale s'applique à la fonction royale; à cet égard, la naapoko royale ne se distingue en rien d'une naapoko ordinaire, à ceci près que la fille aînée du roi défunt ne reste en fonction que sept jours, sauf incident, alors que dans les villages, la durée de l'intérim n'est pas déterminée par la tradition, mais par le contexte dans lequel s'inscrit le règlement politique de la succession. Il est clair que la naapoko intervient comme doublure du naaba: ceci est vrai en général et donc pour la naapoko royale, qui est une doublure du roi-naaba. Tout aussi clairement, l'intérim de la naapoko, qui se termine au moment où est nommé le successeur du chef ou du roi défunt, permet de souder l'une avec l'autre deux périodes de commandement local ou royal, et donc d'inverser en une continuité la discontinuité dans la détention du naam introduite par la mort d'un chef ou du roi. Considérons seulement le cas du pouvoir royal. La suite: ancien règne effectif (a) / prolongement post mortem du règne (sept jours) (b) / intérim de la naapoko (sept jours) (c) / nouveau règne (d) se réduit à l'ajointement: ancien règne $(\mathrm{a}+\mathrm{b}+\mathrm{c}) /$ nouveau règne $(\mathrm{d})$. Ce qui est en cause ici, c'est, à l'évidence, le principe de la continuité du naam . Comme on l'a vu à propos de la généalogie dynastique, qui ne retient que les rimnamba, le réseau de la transmission du naam fait trame pour l'irruption singulière du rinaam. Cette continuité du naam s'inscrit dans l'espace comme immobilité; la naapoko est un personnage qui vit dans l'enceinte du palais royal, là où son père a vécu; la théâtralité de l'exercice de sa fonction n'exige aucun déplacement. 
Avant cette journée, on a choisi parmi les plus jeunes fils du roi défunt - donc parmi ceux qui, en raison de leur rang de naissance, n'auraient aucune chance d'être personnellement concernés par les futures compétitions pour le trône -, celui qui va jouer pendant toute la durée du règne à venir le rôle d'une doublure post mortem de l'ancien roi: son kurita. Le terme kurita est construit sur le modèle de naarita, qui désigne le souverain - ou tout chef - en place, rita étant, comme on l'a vu, un dérivé du verbe ri. Le naarita, c'est le "roi (vivant) régnant", tandis que le kurita, où ku vient du substantif kum, "cadavre", peut être rendu par "(roi) mort régnant". A l'intérieur de l'enceinte du palais, le kurita est revêtu de vêtements royaux et placé sur le premier cheval du roi, le tulubere weefo, dont il sera question plus loin. Le palais, ellipse de grand axe est-ouest, a deux ouvertures, l'une à l'est, l'autre à l'ouest. Chez les Moose, le parcours de la destinée humaine est métaphorisé en un cheminement sur un axe est-ouest: toute initialisation positive est un départ vers l'ouest; à l'inverse, le cheminement ouest-est est associé à la mort. Si le kurita venait à sortir du palais par la porte occidentale, il deviendrait ipso facto rima, du seul fait qu'il est monté sur le tulubere weefo, comme l'est devenu le kurita du fondateur de la dynastie, à une époque où la fonction allait au fils aîné du roi défunt: l'usurpateur est devenu roi sous le "nom de guerre" (zab yuure) ${ }^{\text {ss }}$ de Naaba Kurita. Franchir la porte occidentale en cette circonstance, ce serait reproduire l'acte qui, le jour du sacre, marque l'entrée en fonction du rima. Des captifs royaux, armés de leurs gourdins, protègent l'issue de l'ouest; sous la surveillance de quelques autres captifs, le kurita franchit la porte orientale et quitte la résidence royale en se dirigeant vers l'est; il est conduit jusqu'au village où il sera assigné à résidence pendant toute la durée du nouveau règne: le kurita ne doit pas rencon-

\footnotetext{
${ }^{15}$ Lors de la cérémonie d'intronisation, tout nouveau chef, y compris le roi, énonce trois devises parmi lesquelles les tambourinaires royaux présents choisissent une devise principale dont est extrait un mot embrayeur qui, précédé du terme naaba, devient le nom individuel de fonction de l'intéressé; chacune des devises comme le mot embrajeur de la devise principale est un zab(re) yuure, "nom de guerre".
} 
trer le successeur de son père. Au cours du voyage qui le mène du palais à son lieu d'exil, le kurita est censé pouvoir s'emparer de ce que bon lui semble, mais les captifs royaux qui l'accompagnent veillent à ce que ce droit de prédation ne soit exercé qu'avec parcimonie. Ce qui retient notre attention ici, c'est l'insistance de tous les informateurs sur ce double aspect de l'éphémère rôle du kurita, dont il n'y a d'ailleurs rien d'autre à dire, car, une fois qu'il est installé dans son village, on ne doit plus en entendre parler.

Le kurita est parti du centre du royaume - la résidence royale pour aller vers l'est: ce voyage sans retour est à l'évidence une oblitération définitive. Le kurita ne se manifeste qu'en "prenant", mais de manière dérisoire, tandis que la naapoko "reçoit", et d'autant plus exclusivement que son pouvoir est effectif et qu'il suscite crainte et respect parce qu'il est réputé être exercé avec autorité et sagesse. La naapoko est du côté du naam, avec un marquage fort qui inverse le marquage faible du naam du roi-naaba défunt de la première semaine, tandis que le kurita est du côté du panga, avec un marquage faible qui inverse le marquage fort de la violence des pâsdemba de cette même première semaine. La seconde partie de la période de jonction entre les deux règnes est marquée par une inversion de ce qui prévalait au cours de la première partie: on a toujours un déséquilibre entre le pouvoir et la force, mais c'est désormais le pouvoir qui l'emporte sur la force.

La mise en parallèle des faits relatifs à la naapoko et au kurita ne se justifie pas seulement par la coïncidence dans le temps et dans l'espace de l'émergence de ces deux figures; elle s'impose en outre par le fait qu'autrefois le kurita était le fils aîné du roi défunt - ce qui doit être mis en rapport avec l'ensemble des déterminations négatives qui, de manière générale, pèsent sur le fils aîné -, de sorte que la paire naapoko-kurita était une paire fille aînée-fils aîné, dont le premier terme est toujours marqué positivement et le second négativement. Cette articulation fille aînée (naa(ba) bi(iga) pu(go) kasenga) - fils aîné (naa(ba) bi(iga) kasenga) est d'autant plus fascinante que ces deux personnages jouent ensemble, dans la première 
des deux semaines que nous considérons, les rôles de metteurs en oeuvre des funérailles royales, particulièrement pour ce qui concerne leurs phases "privées", celle de l'inhumation notamment. Le roi-rima est en position de "père" par rapport à la napooko et au kurita; en lui, le naam et le panga sont totalisés sous la forme du rinaam; le roi n'acquiert pleinement sa souveraineté qu'au terme d'un parcours, qui s'ouvre sur un départ vers l'ouest. Ce qui vient d'être dit peut être résumé par le tableau suivant:

$\begin{array}{lll}\text { rima } & \text { naapoko } & \text { kurita } \\ \text { père } & \text { fille aînée } & \text { (fils aîné) } \\ \text { rinaam } & \text { naam } & \text { panga } \\ \text { mobilité } & \text { immobilité } & \text { mobilité } \\ \text { ouest } & \text { centre } & \text { est }\end{array}$

Au moment où il entreprend de faire le ringu, le roi part du centre du royaume et se dirige vers l'ouest, le terme extrême de son voyage étant l'ancienne résidence de Naaba Yaadga, où se déroule le sacre. A son départ, le roi n'est pas encore pangsoba, à son arrivée dans la localité du sacre, il l'est devenu ou est en situation de le devenir. La pauvre épopée du kurita nous indique que la "force" est susceptible d'être affectée par un processus de déperdition, ce qui signifie qu'à l'inverse son acquisition peut relever d'un processus cumulatif. Nous faisons l'hypothèse que l'itinérance du "pouvoir" vers l'ouest produit de la "force", tandis que son itinérance vers l'est la réduit à rien. A la fin d'un règne de rima, au centre du royaume, la configuration naam-panga-rinaam serait soumise à une double procédure de déconstruction: la première séparerait le rinaam des deux autres déterminations, la seconde opérerait une disjonetion entre le pouvoir et la force, dont l'effet est l'oblitération du pouvoir royal résiduel. On a les deux séries d'événements successives (début, fin de règne) suivantes:

(1) naam $\wedge$ panga $(0 \rightarrow 1) \rightarrow$ rinaam
(2) naam $\vee$ panga $(1 \rightarrow 0) \rightarrow$ rinaam $^{(-1)}$.

Ce résultat, qui n'est évidemment consistant qu'avec nos hypothèses de départ, et donc ne correspond qu'à un énoncé analytique, 
introduit la notion d'une intangibilité du naam, qui aurait pour caractère fondamental de demeurer toujours identique à lui-même.

Revenons au voyage du kurita. Les captifs royaux qui l'ont accompagné le laissent dans son village et partent avec le tulubere weefo, qu'ils conduisent à Tangazugu, là où a eu lieu le sacre, pour l'y mettre à mort. Ce cheval, qui est "intronisé" au cours du ringu, doit son nom à l'ornement de chanfrein en cuivre (tulubere) qu'il porte lorsque le roi le monte; c'est un étalon (wee (fo) d raogo, "cheval mâle"), animal emblématique des Moose, dont la devise patronymique (sondre) a pour embrayeur ce même mot wedraogo, qui rappelle le "nom de guerre" du premier des chefs moose, Naaba Wedraogo. Le tulubere weefo ne fait pas de saillies; cette relation négative à l'engendrement n'est pas étrangère à la fonction royale: avant d'accéder au rang de rima, le roi, au cours de la dernière étape du ringu avant le sacre, passe avec celle de ses épouses héritées de son prédécesseur qui va devenir sa principale épouse coutumière sous le nom de rim( $a$ ) poko, "roi-femme", une nuit dont il est de très mauvais présage pour le règne qu'une grossesse s'ensuive, le rima et la rimpoko ne devant plus par la suite entretenir de relations sexuelles ${ }^{16}$. Le tulubere weefo a une aire de

\footnotetext{
in La rimpoko est une épouse en âge de procréer héritée du précédent roi, à laquelle sont assignées d'importantes tâches coutumières, de sorte qu'elle joue le rôle d'une sorte de "reine" officielle, en général sans grande influence sur les affaires du palais, dont est principalement en charge la "reine" officieuse ou rumde. La rimpoko est la gardienne des seuils du palais et du "sanctuaire des ancêtres" ( des trois porteurs des regalia, qui portent les nons de ceux-ci: Wubri, Tiido, Gango; c'est elle qui prépare la bière cérémonielle du roi, qu'elle consonme avec lui, à l'exclusion de tout autre personne; elle fait l'objet d'une salutation distincte de celles qu'on adresse aux autres épouses royales, rumde comprise; ses prérogatives concernent notamment l'accueil au palais des dignitaires de la terre. Du très complexe ensemble de faits relatifs à la rimpoko, nous $n^{\prime}$ avons principalement retenu jusqu'a présent que l'articulation entre la nuit "stérile" du rinqu passée avec le roi, la conception d'un roi sans descendance, donc protégé de tout risque de déperdition de "force", et la "stérilité" imposée au tulubere weefo. Il nous est apparu que la "paire" rima-rimpoko n'était pas un couple mari-ferme, mais manifestait la co-présence au palais d'un principe masculin et d'un principe féminin du rinaam, interprétable dans les termes d'une relation frère-soeur. Dans une correspondance riche en remarques stimulantes faites à la suite de la lecture d'une première version de cet article, Luc de Heusch note que l'unique union du futur rima et de la future rimpoko (le sacre intervient à l'étape suivante du rinqu) évoque tout autant une relation fils-mère qu'une relation frère-soeur, l'une comme l'autre pouvant être marquée par une phase
} 
stabulation abritée qui lui est réservée au palais; il est soigné par des palefreniers particuliers; le roi seul le monte, en certaines grandes occasions. Le roi et son premier cheval sont les seuls "mâles" à vivre dans le palais, dont les autres habitants sont des femmes, des jeunes filles, des garçons non circoncis, des animaux femelles ou castrés. Tout indique que le tulubere weefo est non pas une doublure du roi - comme la naapoko ou le kurita -, mais son double en tant que rima. La mise à mort du tulubere weefo à Tangazugu parachève "l'anti"-ringu dont le moment initial a correspondu à la double nomination de la naapoko et du kurita. Si le tulubere weefo est un double du roi et si on le tue, alors on tue le roi, ce que rappelle l'unité de lieu de ces deux manifestations: l'intronisation du nouveau rima, la mise à mort ignominieuse de son double, dans un univers où la notion de mise à mort rituelle des chefs au terme d'un temps déterminé de commandement est véhiculée par les représentations, à défaut d'être attestée dans les institutions.

incestueuse qui ne doit pas laisser de trace sous la forme d'une naissance qui s'ensuivrait. Si l'hypothèse d'une relation frère-soeur est à coup sûr mal établie, celle d'une relation fils-mère paraît plus difficile encore à valider: 1) la rimpoko est bien une "mère", mais pour les trois porteurs de regalia, les tiidnamba, qui l'appellent bien "notre mère" (tond(o) ma), or on ne peut établir aucune équivalence entre le roi et les tiidnamba; 2) en tant que gardienne du kiimsroogo personnel du roi, elle participe de son ancestralité agnatique, ce qui est consistant avec un statut de soeur, mais non de mère; 3) les Moose pratiquent 1 'héritage des épouses des frères aînés et des "pères", dont le père géniteur, mais, dans ce dernier cas, à l'exception des "mères": mère génitirice, mère "éducatrice", éventuellement "mère" de l'adolescence, embrayeuse d'alliance. Le problème se complique encore du fait qu'il n'y a pas nécessairement une rimpoko par rina: une rimpoko demeure en place aussi longtemps qu'elle peut satisfaire aux conditions de fécondité imposées par le rituel du rinqu: à une seule rimpoko peut donc correspondre plus d'un rima (cas d'un règne ou d'une succession de règne de courte durée). La rimpoko est bien une épouse héritée du précédent roi, mais celui-ci peut, come on dit, l'avoir lui-mêne "trouvée dans la cour". Ce dernier fait invalide d'ailleurs à la fois les hypothèses frère-soeur et fils-mère: la rimpoko, qui n'est pas remplacée avant le rinqu sui vant si elle meurt en cours de règne, relèverait d'un cycle de "rèynes" indépendant de celui des rois; la présence dans le palais de la rimpoko aurait ainsi un caractère de permanence, qui fait peut-être système avec la féminisation générale de l'enceinte royale, seulement contredite par la double présence du roi et du tulubere weefo. Il y a là tout un dossier à réexaminer. 
Nous pouvons maintenant proposer une interprétation de ce qu'est entre autres choses le ringu. Durant la première partie de ce voyage, le naaba, qui se dirige vers l'ouest, acquiert progressivement le statut de pangsoba. Au jour du sacre, il y a conjonction naam-panga et instauration du rinaam, marquée par l'apparition du roi monté sur le tulubere weefo, alors que pendant la première partie de son voyage, le roi est apparu à pied devant les autels de la terre, puis monté sur un âne lorsqu'il a gravi la colline de Tangazugu, au sommet de laquelle se trouvent l'ancienne demeure de Naaba Yaadga et la "pierre du pouvoir". Le "pouvoir" et la "force" ont fait du roi un rima, et c'est la mise en relation du "pouvoir" et de la "terre" par le moyen de sacrifices faits sur les autels de la terre des "villages des ancêtres" des rois qui a produit la "force".

Résumons-nous. 1 - Le roi est inhumé dans le cimetière royal; cette inhumation concerne la personne du souverain en tant qu'elle existe indépendemment de toute détermination singulière liée à la détention du rinaam, du panga ou du naam; elle transforme le roi en ancêtre bienveillant à l'égard de son successeur. 2 - Par le truchement de la naapoko, qui représente son père en tant que roi-naaba, la continuité de la transmission du naam redevient prévalente sur la nécessaire discontinuité introduite par le règne qui vient de s'achever; on peut parler d'une résorption dans la durée, celle du temps dynastique. 3 - Par le truchement du kurita, c'est, comme l'indique clairement l'emphase portée sur le droit de prédation du prince, la trace du roi-pangsoba qui est abolie. L'expulsion du kurita, à l'instar de la procédure générale du bannissement, a valeur de résorption dans l'étendue, celle de l'espace du royaume. En partant du centre du royaume pour se perdre vers l'est, le kurita accomplit un voyage qui est symétrique et inverse de celui que le roi-naaba, en quête de "force", accomplit au cours de la première partie du ringu en partant du même centre mais en se dirigeant vers l'ouest. 4 - La mise à mort du tulubere weefo referme le règne sur lui-même en abolissant la présence au monde du rima là même où celle-ci s'était d'abord manifestée. 
La relation entre rinaam, panga et naam, et, parallèlement, entre les figures du rima, du pangsoba et du rima, commence a mieux nous apparaître, et avec elle la conception mooga de la double valence du rima, à laquelle renvoient la dualité pangsoba-naaba et en amont la dualité panga-naam. La triple détermination rima/pangsoba/naaba rend compte du parachèvement de la figure du souverain; le rima n'est pas réductible à la complémentarité pangsoba-naaba, il est autre chose et plus que la totalisation de ces deux figures primitives.

La structure ternaire:

naaba/pangsoba/rima

n'est pas sans évoquer celles, homologues entre elles, qui ont été rappelées ci-dessus.

Le parigsoba nous est apparu comme étant un avatar du rima lié à l'étendue, la naaba en étant un avatar lié à la durée; il y a homologie entre les relations:

rima/pangsoba

et

personne/génie;

ainsi qu'entre les relations:

rima/naaba

et

homme/ancêtre.

Nous faisons 1'hypothèse que la configuration:

rima/pangsoba/naaba

relève du même encodage général que nos autres triades, ce qui constitue, entre autres, le roi pleinement roi - le rima -, comme une "expansion" remarquable de la personne humaine en cause, en ce que l'étendue concernée est l'espace fermé du royaume et la durée, celle de l'ancestralité dynastique. Il existerait ainsi un rapport général à la fois homologique et homothétique entre les personnes ordinaires et 
la personne royale, le propre de la personne ordinaire étant que les déterminations à travers lesquelles elle se constitue relèvent de catégories abstraites (quels que soient les référents "réels" auxquels celles-ci renvoient ici et maintenant pour telle personne dûment identifiée), tandis que la personne royale relèverait de déterminations d'ordre territorial et historique, dûment "traitées" par les rituels de sou veraineté pour être bonnes à penser symboliquement, non seulement par les détenteurs du pouvoir, mais aussi par ceux -les autochtonesauxquels ce pouvoir s'est imposé, précisément par la force. Le roi serait, dans le royaume, le seul personnage à disposer de deux personnalités entre lesquelles nous pouvons établir une relation d'homologie: sa personne ordinaire et sa "personne" royale. Nous avons rappelé ci-dessus la place cruciale que nous assignons à la triade: identité/êtendue/durée, qui rend compte du statut ontologique de l'être humain; cette double relation peut s'écrire ainsi:

(1) personne/étendue/durée;

Si nous considérons la personne remarquable qu'est le roi et les avatars des paramètres qui en définissent l'existence, on a, homologue de la précédene, la double relation:

(2) roi/étendue du royaume/durée de la dynastie.

Ce qui précède conduit à reconsidérer l'articulation entre le pouvoir et ses deux instances de légitimation, dont rend compte l'écriture:

pouvoir/autochtonie/ancestralité.

Nous avons initialement fait de cette double relation un produit de déconstruction de la notion de pouvoir-naam; voici maintenant que le pouvoir-naam nous apparaît comme étant lui-même l'un des éléments de décons truction de la notion de pouvoir-rinaam, ce que nous exprimons en donnant à la double relation:

$$
\text { rima/pangsoba/naaba (cf.ci-dessus) }
$$

l'équivalent:

rinaam/panga/naam. 
D'où cette nouvelle hypothèse: le naam a pour déterminants généraux l'autochtonie et l'ancestralité, le panga (force) et le naam (pouvoir) étant au rinaam (souveraîneté) ce que l'autochtonie et l'ancestralité sont au naam (pouvoir). On a une itération qui peut être développée ainsi:

(1) ancestralité/autochtonie/pouvoir

(2) pouvoir/force/souveraineté

ce qui peut s'écrire de la manière suivante, qui permet de mieux mettre en évidence l'existence de deux paliers de "pouvoir":

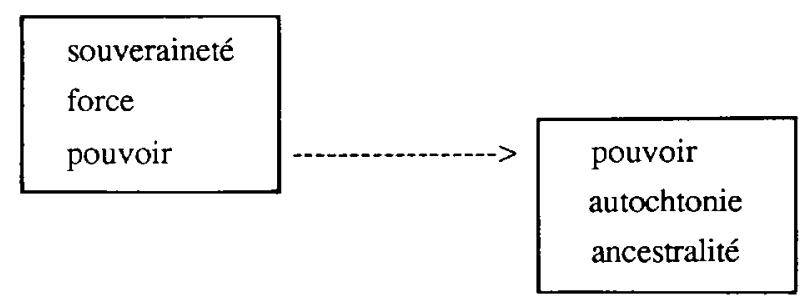

Pour ce qui concerne la construction de la souveraineté, le ringu équivaut à une intrusion de la "force" dans la sphère du "pouvoir". En d'autres termes, la relation du panga à l'étendue permet d'envisager le sacre du rima comme une véritable naissance, l'émergence de la "force", née du monde des génies, valant individuation: où nous retrouvons le théorie mooga de l'élaboration de la personne par intervention des génies comme opération de partition du flux de substance ancestrale. A la mort du rima, ce qu'il y a de génie en lui est expulsé sous la forme du kurita. Le contenu rituel des manifestations du ringu avant le moment du sacre conduit à considérer que l'émergence de la force n'est possible que par la médiation de la "terre". Nous sommes là dans le champ de l'autochtonie; la "force" naît de sacrifices faits sur les autels de la terre des anciennes résidences royales. Est-ce à dire que ces autels sont producteurs de cette "force" que le rima va capter? On constatera en tout cas que la relation institutionnelle à la force et à la terre est exprimée par le même verbe, so, dont est dérivé le substantif soba, présent à la fois dans teng soba 
et dans pang soba": l'ordre des sobnamba (plur. de soba) et celui des nanamba (plur. de naaba) s'opposent comme, par ailleurs, l'instance de l'autochtonie et celle de l'ancestralité, à partir desquelles se constitue toute personne: celle du roi, radicalement autre, et celle de n'importe qui'".

\section{Michel Izard}

Laboratoire d'Anthropologie sociale

\footnotetext{
17 A teng soba, maître de la terre, et pang soba, maître de la force, il faut ajouter, pour ce qui concerne la titulature fondée sur la substantivation de so en soba, tâ soba, maître de la guerre: l'ensemble de la sémantique de so, l'action, soba, l'agent, et solem, l'objet, reste dans une large mesure à analyser.

${ }^{18} \mathrm{~L}$ 'opposition naaba/soba est à mettre en rapport avec une opposition entre ce sur quoi s'exerce le pouvoir, à savoir les hormes ou le monde humain, et ce qui relève du type d'intervention que rend le verbe so, ainsi, on l'a vu: la terre, la force et la guerre. Il est possible, à cet égard, que traduire so par "avoir la maîtrise (de)" puisse prêter à confusion: avoir la maitrise (de) n'est pas "commander (à)", c'est disposer des moyens d'un contrôle de la manifestation de forces que l'homme, précisément, ne maîtrise pas. On est conduit à faire l'hypothèse - la valider est une autre affaire - que ce que nous appelons la "terre" - à laquelle sont associés des phénomènes tels que le vent, la pluie, la foudre, etc. - a un statut voisin de la "nature", ce qui, par delà le dualisme "divin" Wende-Tenga, pose le problème de ce qu'est en vérité le monothéisme mooga. Au contraire d'une "maîtrise" de la nature, au sens, par exemple, hégélien du terme, qui serait une domination de 1 'homme sur la nature, on aurait un rapport de domination de la nature sur 1 'home; la nature pouvant se mani fester corme favorable ou défavorable, dangereuse ou bienveillante, 1 'home disposerait des moyens (essentiellement rituels) d'infléchir les effets de cette domination dans un sens bénéfique, sous réserve que cette adéquation des manifestations de la nature aux visées humaines ait pour contrapartie une mise en conformi té de l'activité humaine à l'éthicité qui justifie la demande faite à la nature, ou l'exigence à laquelle elle est soumise.
} 


\section{Références}

Izard M. ,

1983 "Engrammes du pouvoir. L'autochtonie et l'ancestralité", Le Temps de la réflexion IV, 299-323.

1985 Gens du pouvoir, gens de la terre. Les institutions politiques de l'ancien royaume du Yatenga (Bassin de la Volta Blanche), Cambridge, Cambridge University Press/ Paris, Editions de la Maison des sciences de l'homme.

1986 "L'étendue, la durée" L'Homme 97-98, L'anthropologie, état des lieux, 225-237, repris in L'Homme. Anthropologie: état des lieux, Paris, Navarin/Le Livre de Poche (Le Livre de Poche, 4046/Biblio-Essais, VII) : 249-262. 